\title{
sciendo
}

Current Issues in Pharmacy and Medical Sciences

Formerly ANNALES UNIVERSITATIS MARIAE CURIE-SKLODOWSKA, SECTIO DDD, PHARMACIA

\section{Obtaining the tbf gene which encodes immunodominant epitopes of pathogenic cholera strains}

\author{
Yulia Bazarnova* ${ }^{\circledR}$, Tatiana Bolotnikova, Ekaterina Aronova
}

Higher School of Biotechnology and Food Technology, Peter the Great Saint Petersburg State Polytechnical University

\section{ARTICLE INFO \\ Received 06 November 2018 \\ Accepted 08 February 2019}

\section{Keywords:}

candidate cholera vaccine,

hybrid tbf gene,

Sanger sequencing,

protein design,

TBF recombinant protein,

immunodominant epitopes,

Vibrio cholerae.

\begin{abstract}
We experimentally carried out the synthesis of the tbf gene that encodes highlyimmunogenic epitopes of pathogenic cholera strains, through the cloning of the tbf gene in pGEM-T Easy plasmid. Moreover, we tested the hybrid gene sequence for absence of mutations, using the Sanger sequencing. We also calculated the nucleatic sequence of the tbf gene. The obtained results have both scientific and practical significance.
\end{abstract}

\section{INTRODUCTION}

Cholera is an intestinal infection disease that rapidly dehydrates the human organism. It is caused by the bacterium of the Vibrio cholerae species, namely, toxigenic serogroup $\mathrm{O} 1$ and less commonly, O139. Cholera is characterized by a fecal-oral transmission route (more rarely humanto-human direct transmission) affecting the small bowel, and symptoms that include watery diarrhea, vomiting, rapid loss of water and electrolytes with various ranges of dehydration up to hypovolemic shock.

The cholera toxin (exotoxin) is responsible for the disease symptoms and its rapid evolution, and is a key factor of its pathogenicity.

As of today, scientists have come to a conclusion that oral immunization is the most efficient immune protection from cholera. Along with the developed attenuated and inactivated oral anti-cholera vaccines, recombinant vaccines based on epitopes of bacterial antigens occupy leadership positions for cholera treatment. These vaccines induce a pronounced immune response and depress the bacterial transcytosis, which is a key factor for the disease evolution.

The object under study in this work is the $t b f$ gene that encodes immunodominant epitopes of proteins of pathogenic cholera strains TcpA and $\mathrm{B}(\mathrm{rBS})$, as well as an area which provides antigen penetration through the epithelium of the stomach wall.

\footnotetext{
* Corresponding author

e-mail: j.bazarnowa2012@yandex.ru
}

The TcpA protein is a component of a toxin-coregulated adhesion pilus, and forms polymers from subunits of $20.5 \mathrm{kDa}$ [1]. This protein is a factor in pathogen colonization [2]. Toxin-coregulated piles are important protective antigens, which possess well-pronounced protective properties [3].

The subunit of cholera exotoxin B (rBS) is a pentamer, each sub-subunit of which is presented as a polypeptide chain consisting of 103 amino acid residues [4]. The protein contains antigenic determinants [5], which induce neutralization antibodies.

The neonatal FcRn-receptor is a receptor of the Fc fragment that forms a heterodimer by non-covalent interaction of alpha-chain with beta-2 microglobulin [6]. At oxygen hydrolysis, the $\mathrm{pH}$ receptor bounds the segment that connects $\mathrm{CH} 2$ - and $\mathrm{CH} 3$-domains of antibodies of the IgG class [7]. After antibody bonding, the receptors activate a cascade of reactions for pathogen elimination by antibodydependent phagocytosis [8].

Thus, the vaccine, which contains purified immunogenic protein, is stable and safe, its chemical properties are wellstudied, and it does not contain any additional proteins and nucleic acids that might cause undesirable effects in the host organism.

The aim of this work is to inform readers of a means of replicating the $t b f$ gene that encodes immunodominant epitopes of pathogenic cholera strains.

The work was performed at Laboratory No. 6 "Genetic engineering of vaccines" of the State Research Institute of 
Highly Pure Biopreparations of the Russian Federal Medical-Biological Agency (FMBA), Saint-Petersburg, Russia.

\section{MATERIALS AND METHODS}

\section{Objects under study}

The gene, which encodes TBF recombinant protein, was obtained through chemical synthesis. Synthesis of sequence of the calculated gene that encodes the TBF protein, was performed by applying the polymerase chain reaction technique, using overlapping oligonucleotides. These oligonucleotides were synthesized by by employing the DNA synthesizer ASM-800 (BIOSSET, Russia). The main requirements for primers were the following: length not more than 60 nucleotides, and hybridization segments not more than 20 nucleotides. Beyond the aforementioned, there should not be long segments with repeated $\mathrm{G}$ or $\mathrm{C}$. In total, for the synthesis of the gene that encodes the TBF protein of 2085 nucleotide pairs, we used 77 primers. The synthesized sequence was obtained from agarose gel, by means of electrophoresis, and cloned in the plasmid vector pGEM-T Easy. We performed the blunt-end cloning. After sequencing utilizing the capillary sequencer Applied Biosystems 3500/3500xL Genetic Analyzer (Applied Biosystems, USA), the fragments were amplified in a thermocycler for amplification - C1000 ThermalCycler (Bio-Rad, USA). In the terminal gene segments, we included restriction sites XhoI and NdeI for further cloning in plasmid pET28a $(+)$.

The vector map is presented in Figure 1.

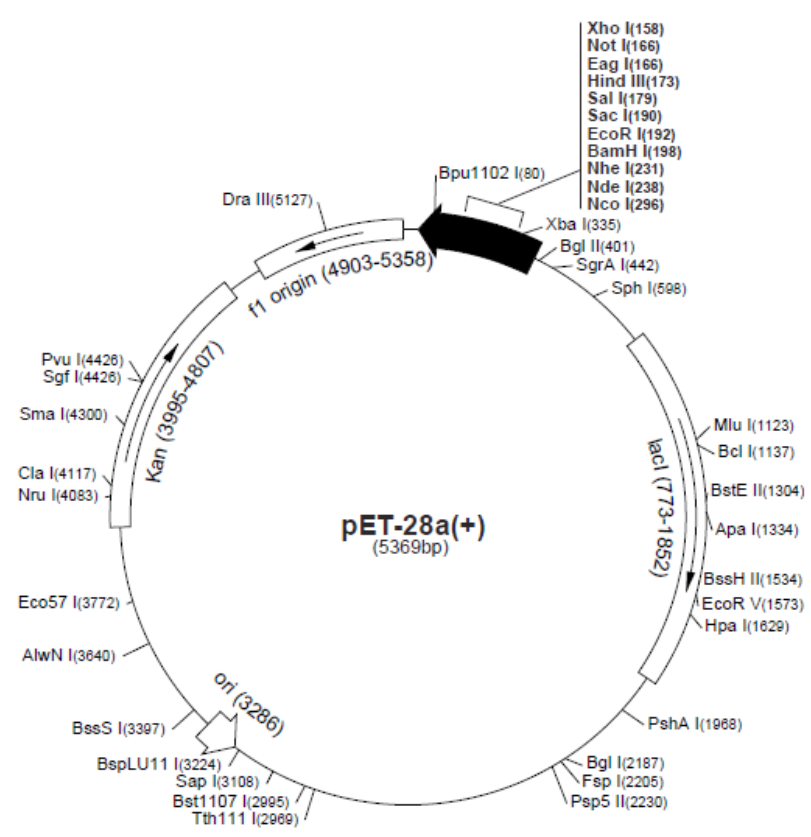

Figure 1. Circular pET28a (+) plasmid map [9]

The expression plasmid comprises the replication origin, promoter of T7 phage polymerase, lac-operator, kanamycinresistance gene, start codon for translation of cloned fragments and a fragment which encodes polyhistidine located in the reading frame at the $\mathrm{N}$-end of the sequence. Hence, any nucleatic sequence cloned in the vector is expressed as a polyhistidine-tagged protein as a matter of convenience for its further purification by means of immobilized metal affinity chromatography. For lac-operon operation, the plasmid contains a fragment that encodes the lactose repressor lacI.

The gene cloning in the vector was performed via the XhoI and NdeI restriction sites.

For the genetic engineering, we used E. coli $\mathrm{DH} 10 \mathrm{~B} / \mathrm{R}$ (Gibko BRL, USA) cells with F-mcrA $\Delta$ (mrr-hsdRMSmcrBC) $\varphi 80$ dlacZ $\Delta \mathrm{M} 15 \Delta$ lacX74 deoR recA1 endA1 $\operatorname{araD139} \Delta$ (ara,leu) 769 galUgalK $\lambda$ - rpsLnupG genotype.

For the expression of the gene that encodes the TBF protein, we employed E.coli BL21 Star (DE3) cells with the F- ompThsdSB (rB-mB-) galdem rne131 (DE3) genotype that contains in its genome, $\lambda$ De 3 lysogen and rne 131 mutation. The mutated gene rne (rne131) encodes reduced RNAase E, which decreases the intracellular destruction of mRNA. This results in the enhancement of its fermentation stability. Of note, lon- and ompT- mutations in protease genes allow the possibility to obtain large amounts of nonproteolyzed recombinant proteins.

\section{Research methods}

Synthesis of sequence of the calculated gene that encodes TBF protein, was performed by means of the polymerase chain reaction technique and the use of overlapping oligonucleotides. These oligonucleotides were synthesized by means of the DNA synthesizer ASM-800 (BIOSSET, Russia). The main requirements for the primers were the following: length not more than 60 nucleotides and hybridization segments not more than 20 nucleotides. Moreover, there should not be long segments with repeated $\mathrm{G}$ or $\mathrm{C}$. In total, for the synthesis of gene that encodes the TBF protein of 2085 nucleotide pairs, we used 77 primers. The synthesized sequence was obtained from agarose gel, through electrophoresis, and cloned in the plasmid vector pGEM-T Easy. We performed the blunt-end cloning. After sequencing via the capillary sequencer Applied Biosystems 3500/3500xL Genetic Analyzer (Applied Biosystems, USA), the fragments were amplified in a thermocycler for amplification C1000 ThermalCycler (Bio-Rad, USA). In the terminal gene segments, we included restriction sites XhoI and NdeI for further cloning in plasmid pET28a(+).

For the design of TBF recombinant protein, we accessed NCBI databases (www.ncbi.nlm.nih.gov).

\section{RESULTS}

For the design of the TBF recombinant protein, we used the most protective epitopes of bacterial antigens: subunit of B cholera exotoxin, protein of cholera A pilus, and domain, which is a ligand to $\mathrm{Fc}$-receptors at the stomach wall. The selected consensus segments, common for $V$. cholerae pathogenic strains, were analyzed for $\mathrm{T}$ and $\mathrm{B}$ cell epitopes. During the analysis, we employed the following software: Bepipred Linear Epitope Prediction 2.0 and IEDB Analysis Resource. The obtained amino acid sequence of TBF recombinant protein (695 amino acid residues) is presented in Figure 2. 


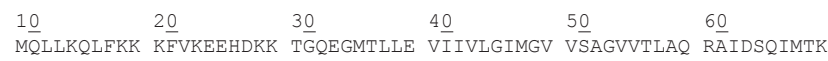

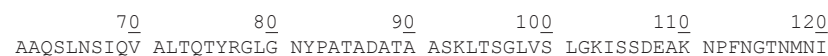
$\begin{array}{llllll}13 \underline{0} & 14 \underline{0} & 15 \underline{0} & 16 \underline{0} & 17 \underline{0} & 18 \underline{0}\end{array}$ FSFPRNAAAN KAFAISVDGL TQAQCKTLIT SVGDMFPYIA IKAGGAVALA DLGDFENSAĀ

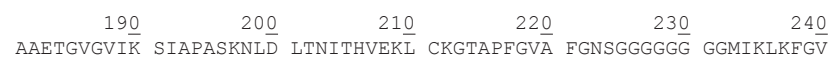
$\begin{array}{llllll}25 \underline{0} & 26 \underline{0} & 270 & 280 & 29 \underline{0} & 30 \underline{0}\end{array}$

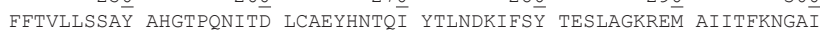

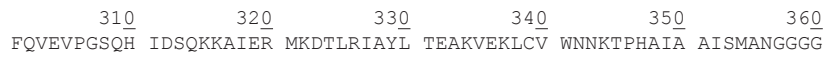

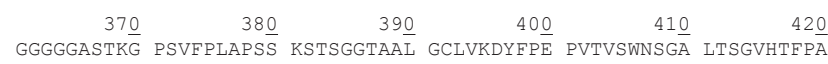

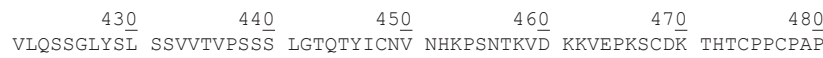

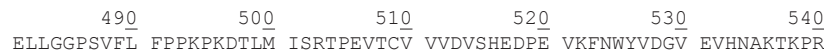

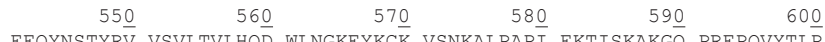
$\begin{array}{llllll}610 & 620 & 630 & 640 & 650 & 660\end{array}$ PSRDELTKNQ VSLTCLVKGE YPSDIAVEWE SNGQPENNYK TTPPVLDSDG SFELYSKLTV 670
DKSRWQGGN $\frac{680}{690}$ FSCSVMHEAL HNHYTQKSLS LSPGK

Figure 2. TBF protein amino acid sequence

The nucleatic sequence that encodes the TBF protein (with length of 2085 nucleotide pairs) was obtained via calculation (Fig. 3).

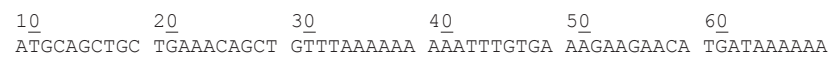

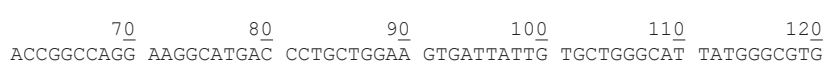

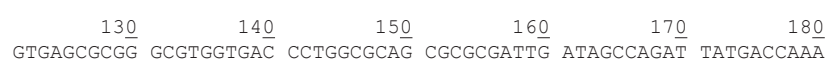

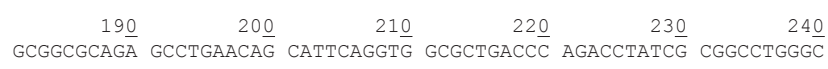
$\begin{array}{llllll}25 \underline{0} & 26 \underline{0} & 27 \underline{0} & 28 \underline{0} & 29 \underline{0} & 30 \underline{0}\end{array}$ AACTATCCGG CGACCGCGGA TGCGACCGCG GCGAGCAAAC TGACCAGCGG CCTGGTGAGC

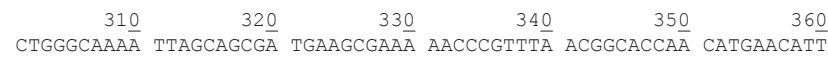
CTGGGCAAAA TIAGCAGCGA TGAAGCGAAA AACCCGITIA ACGGCACCAA CATGAACATI

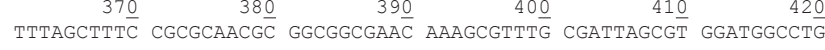

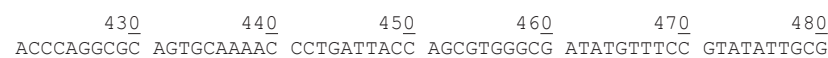

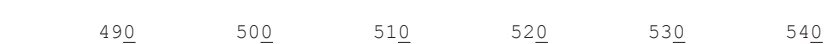

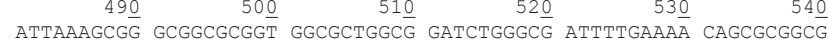

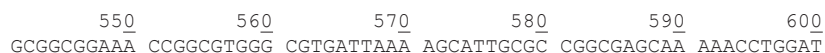
$\begin{array}{rrrrr}610 & 620 & 630 & 640 & 650\end{array}$

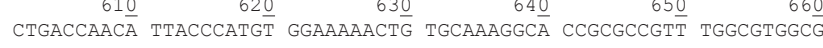

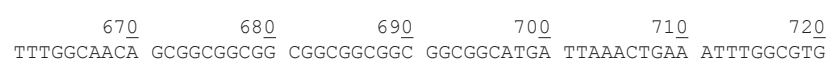
$\begin{array}{llllll}73 \underline{0} & 74 \underline{0} & 75 \underline{0} & 76 \underline{0} & 77 \underline{0} & 78 \underline{0}\end{array}$

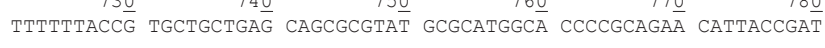

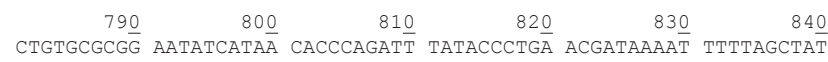

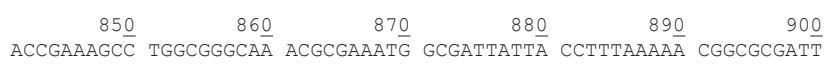

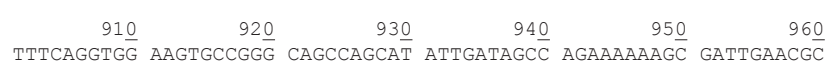

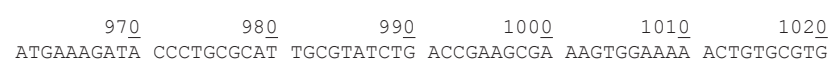
$\begin{array}{llllll}103 \underline{0} & 104 \underline{0} & 105 \underline{0} & 106 \underline{0} & 107 \underline{0} & 108 \underline{0}\end{array}$ TGGAACAACA AAACCCCGCA TGCGATTGCG GCGATtAgCA TGGCGAACG $\overline{0}$ CGGCGGCGG

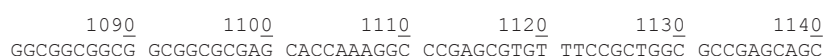

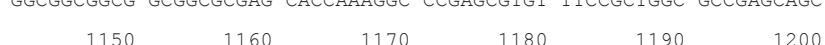

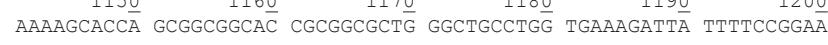

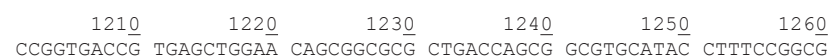
$\begin{array}{llllll}127 \underline{0} & 128 \underline{0} & 129 \underline{0} & 130 \underline{0} & 131 \underline{0} & 132 \underline{0}\end{array}$ GTGCTGCAGA GCAGCGGCCT GTATAGCCTG AGCAGCGTGG TGACCGTGCD GAGCAGCAGC $\begin{array}{rrrrrr}1330 & 1340 & 1350 & 1360 & 1370 & 1380 \\ \text { CTGGGCACCC } & \text { AGACCTATAT } & \text { TtGCAACGTG } & \text { AACCATAAAC } & \text { CGAGCAACAC } & \text { CAAAGTGGAT }\end{array}$

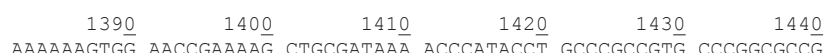

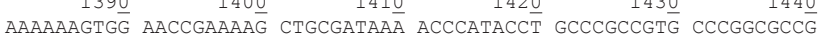

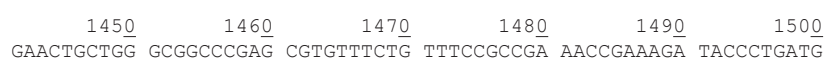
$\begin{array}{llllll}151 \underline{0} & 152 \underline{0} & 153 \underline{0} & 154 \underline{0} & 155 \underline{0} & 156 \underline{0}\end{array}$

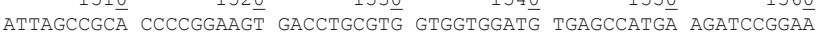
$\begin{array}{rrrrrr}157 \underline{0} & 158 \underline{0} & 159 \underline{0} & 160 \underline{0} & 161 \underline{0} & 162 \underline{0}\end{array}$ GTGAAATTTA ACTGGTATGT GGATGGCGTG GAAGTGCATA ACGCGAAAA $\overline{\bar{C}}$ CAAACCGCGC $\begin{array}{rrrrrr}1630 & 1640 & 1650 & 1660 & 1670 & 1680 \\ \text { GAAGAACAGT } & \text { ATAACAGCAC } & \text { CTATCGCGTG } & \text { GTGAGCGTGC } & \text { TGACCGTGCT } & \text { GCATCAGGAT }\end{array}$ $\begin{array}{llllll}169 \underline{0} & 170 \underline{0} & 171 \underline{0} & 172 \underline{0} & 173 \underline{0} & 174 \underline{0}\end{array}$

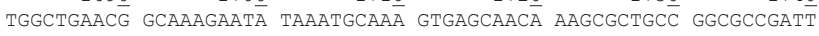

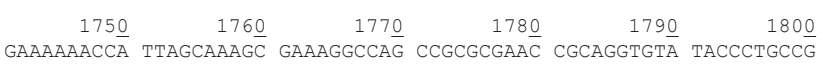
$\begin{array}{llllll}181 \underline{0} & 182 \underline{0} & 183 \underline{0} & 184 \underline{0} & 185 \underline{0} & 186 \underline{0}\end{array}$

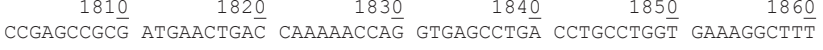
$\begin{array}{llllll}187 \underline{0} & 188 \underline{0} & 189 \underline{0} & 190 \underline{0} & 191 \underline{0} & 192 \underline{0}\end{array}$

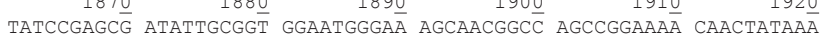

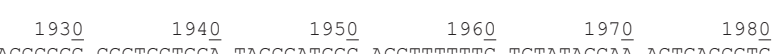

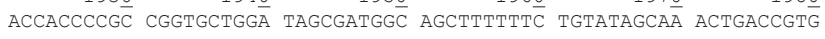
$\begin{array}{rrrrr}1990 & 2000 & 2010 & 2020 & 2030\end{array}$

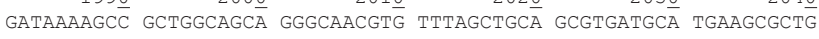
$\begin{array}{rrrr}2050 & 2060 & 2070 & 2080 \\ \text { CATAACCATT } & \text { ATACCCAGA } \bar{A} & \text { AAGCCTGAGC } & \text { CTGAGCCCGG } \\ \text { GCAAA }\end{array}$

Figure 3. tbf gene nucleatic sequence

\section{DISCUSSION}

In the study, we carried out the synthesis of a calculation model of tbf gene sequences with length of 2085 nucleotide pairs by means of the PCR technique and overlapping oligonucleotides that were synthesized via the DNA synthesizer ASM-800 (BIOSSET, Russia). The absence of mutations in the sequence of hybrid gene was indicated through applying the sequencing technique (Fig. 4).

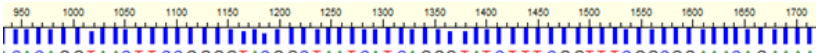

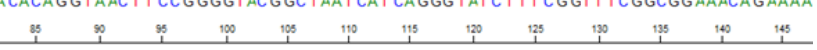

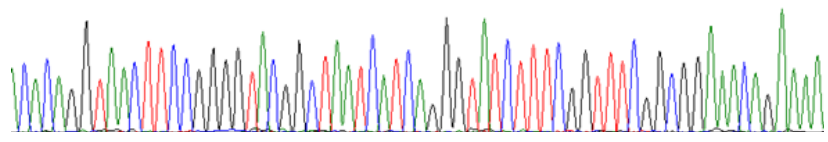

W1111000

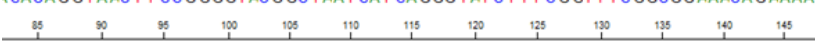

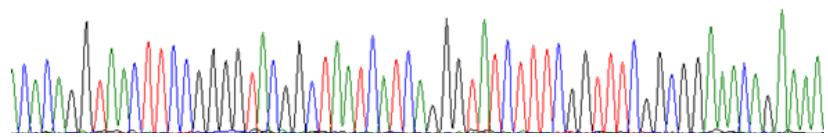

Figure 4. A tbf gene chromatogram fragment. Interpretation according to the direct-primer Sanger technique (TAATACGACTCACTATAGGG)

Sequencing of amplified DNA segments was performed according to the Sanger technique.

\section{CONCLUSIONS}

Our study was the first to calculate the sequence of nucleotide pairs forming part of $t b f$ gene that encodes immunodominant epitopes of pathogenic cholera strains and to synthesized it.

We cloned the $t b f$ gene in plasmid pGEM-T Easy and examined the hybrid gene sequence for the absence of mutations, using the Sanger sequencing technique. 
The obtained results are of interest for further development of stable and safe recombinant vaccines that contains subunits of cholera toxin B (rBS), protein of cholera A piles (TcpA), as well as the domain that is a ligand to Fc-receptors at the stomach wall $(\mathrm{FcL})$.

\section{ORCID iDs}

Julia Bazarnova (Dhttps://orcid.org/0000-0001-9275-7913

\section{REFERENCES}

1. Kiaie S, Abtahi H, Mosayebi G, Alikhani M, Pakzad I. Recombinant toxin-coregulated pilus A (TcpA) as a candidate subunit cholera vaccine. Iran J Microbiol. 2014;6:68-73.

2. Megli CJ, Yuen AS, Kolappan S, Richardson MR, Dharmasena MN. Crystal structure of the Vibrio cholerae colonization factor TcpF and identification of a functional immunogenic site. Mol Biol. 2011;409:146-58.
3. Liljeqvist S. Surface display of the cholera toxin B subunit on Staphylococcus xylosus and Staphylococcus carnosus. Appl Environ Microbiol. 1997;63:2481-8.

4. Sanchez J, Johansson S, Löwenadler B, Svennerholm A, Holmgren J. Recombinant cholera toxin B subunit and gene fusion proteins for oral vaccination. Research in Microbiology. 1990;141:971-9.

5. Praetor A, Jones RM, Wong WL, Hunziker W. Membrane-anchored human FcRn can oligomerize in the absence of IgG. J. Mol. Biol. 2002;321:277-84.

6. Schroeder HW, Cavacini L. Structure and function of immunoglobulins. J Allergy Clin Immunol. 2010:125(2,Suppl 2):S41-52. https://doi.org:10.1016/j.jaci.2009.09.046

7. Raghavan M, Bjorkman PJ. Fc receptors and their interactions with immunoglobulins. Annu Rev Cell Dev Biol. 1996;12:181-220.

8. Reed SM, Bayly WM, Sellon DC. Equine internal medicine (4 edition). St. Louis, Missouri: Elsevier;2018:1566.

9. Addgene: pET28a(+) www.addgene.org/20378/ . 